\title{
Les technologies du sujet
}

Une approche ethno-philosophique

Technologies of the Subject: an Ethno-Philosophical Approach

Jean-Pierre Warnier

\section{(2) OpenEdition}

Journals

Édition électronique

URL : https://journals.openedition.org/tc/4853

DOI : $10.4000 /$ tc. 4853

ISSN : 1952-420X

Éditeur

Éditions de l'EHESS

\section{Édition imprimée}

Date de publication : 1 décembre 2009

Pagination : 148-167

ISBN : 978-2-7351-1301-9

ISSN : 0248-6016

Référence électronique

Jean-Pierre Warnier, "Les technologies du sujet », Techniques \& Culture [En ligne], 52-53 | 2009,

document 5, mis en ligne le 01 août 2012, consulté le 29 septembre 2022. URL : http://

journals.openedition.org/tc/4853; DOI : https://doi.org/10.4000/tc.4853 


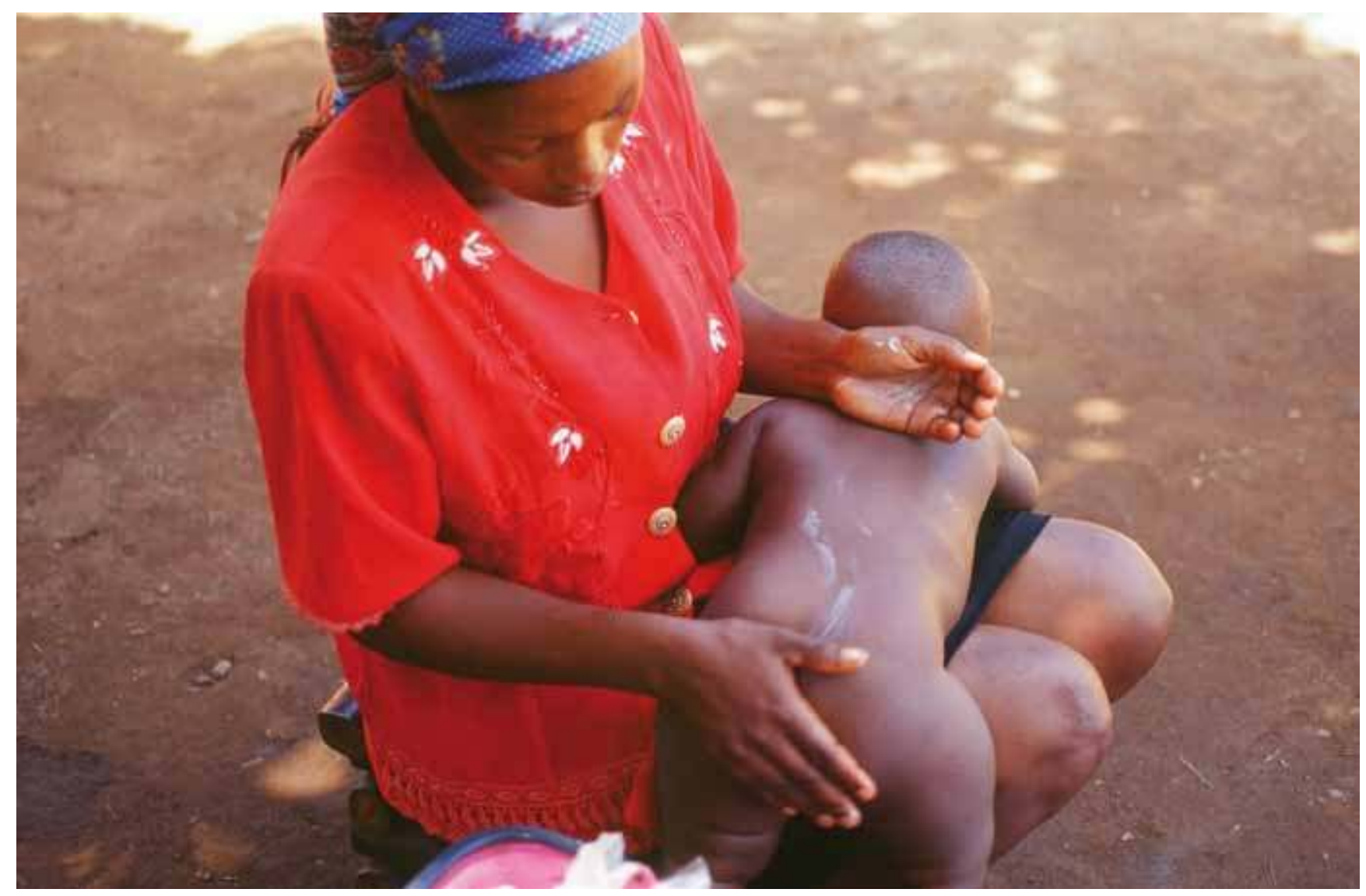




\section{LES TECHNOLOGIES DU SUJET}

\section{Une approche ethno-philosophique}

Seule la notion de sujet, à l'exclusion de la notion d'individu, est à même d'intégrer à titre essentiel le corps, les investissements libidinaux, l'inconscient comme refoulé, la culture matérielle, les kinesthèses, l'appareil sensoriel et l'intersubjectivité. Elle permet d'établir un rapport d'immanence entre le sujet, la technologie et la culture matérielle.

La technologie, comme savoir sur les techniques, s'applique non seulement aux matières et artefacts, mais également aux sujets et à leur corps. Il existe des techniques du corps et du sujet qui ont pour résultat de les produire, de les configurer, d'en faire usage, et de les recycler. Ces techniques du corps et du sujet sont largement médiatisées par l'usage et l'incorporation/ désincorporation de la culture matérielle dans le mouvement.

Je déploierai le contenu de ces idées liminaires en commentant les mots et expressions fondamentales qui y figurent. Cela me permettra de situer cette approche ethno-philosophique des technologies du sujet tant par rapport à la technologie culturelle à la française que par rapport aux études de culture matérielle pratiquées outre-Manche autour de la consommation ou des objets en tant qu'indicateurs ou signes. 


\section{Corps et techniques du corps}

Qu'est-ce qu'un corps? Le corps n'est constitué comme tel qu'en étant investi par un sujet qui est et qui a un corps. Si le sujet n'est pas constitué, le corps en est réduit à quelques dizaines de kilogrammes d'organes qui ne sont traversés d'aucune intentionnalité. Je reviendrai plus tard sur ce rapport entre « corps » et « sujet », essentiel à mon propos. À la suite de Mauss (1936), on peut définir les « techniques du corps » comme « les manières qu'ont les humains, société par société, de se servir de leur corps». Je glose cette définition en disant que les techniques du corps s'appliquent au corps en tant qu'objet d'une action technique (pour le produire, le configurer, etc.) et qu'elles font usage de celui-ci à titre d'outil d'action sur le corps du sujet ou d'un autre sujet. C'est, écrit Mauss, «le premier de tous les outils $»$. Faute de place, je me permets de renvoyer le lecteur à un commentaire du texte de Mauss que j'ai publié ailleurs (Warnier 1999 : 21-36).

Sur le corps, il existe une abondante littérature, y compris deux dictionnaires - ceux d'Andrieu (2006) et de Marzano (2007). Dans cette littérature, le meilleur jouxte le pire. Lanthropologie phénoménologique qui s'est développée à partir des années 1980 - celle de Le Breton (1990), Csordas (1994) et Featherstone (\& al. Eds., 1991) par exemple-eut le mérite de prendre la question «à bras-le-corps ». Mais elle est insatisfaisante en ce qu'elle s'est distancée de Husserl et qu'elle est

Soutirage de la sève de raphia

La question est de caractériser le complexe constitué par le sujet, son corps et ses objets dans l'action (Mankon, 2002). solidaire d'une philosophie du sujet calée sur le seul cogito. Berthelot (1988), Berthoz et Petit (2006), l'œuvre de Foucault et les travaux du séminaire de Dominique Memmi et Florence Bellivier « Corps et sciences sociales », offrent des bases beaucoup plus solides pour une approche rigoureuse $\mathrm{du}$ « corps ».

\section{Sujet, subjectivité, technologies du sujet}

Pour aller à l'essentiel, on peut structurer le débat sur le sujet autour des figures emblématiques de Husserl ${ }^{2}$ et de Sartre. Husserl fait sa place au corps puisqu'il considère le corps comme le truchement de l'être-au-monde 
et de la constitution de son monde par le sujet. Avec le corps, il fait sa place à tout ce que le sujet a d'obscur et qui le fait participer à ce que Hegel nommait « la nuit du monde ». Sartre, par contre, purifie le sujet de tout ce qui l'empêcherait d'être une conscience claire, calée sur le cogito, transparente à elle-même, vouée à l'engagement et à l'exercice de la liberté. Telle est la raison du rejet de la psychanalyse par Sartre. Si le sujet est celui du cogito, pense Sartre, Freud se trompe.

Foucault, on le sait, fait le raisonnement inverse: si Freud a raison dit-il, la philosophie du sujet doit être repensée, « autrement ». Il rejette le sujet sartrien qui ne tient pas compte de la découverte freudienne de l'inconscient comme refoulé, ni de l'apport des sciences de l'homme et de la société qui illustre toutes les dimensions de l'existence humaine échappant à une prise de conscience claire par le sujet. Deleuze, Certeau et d'autres furent également très présents dans ce débat. Celui-ci peut paraître ésotérique. En fait, il est central à mon propos, car la question est de caractériser le complexe constitué par le sujet, son corps et ses objets dans l'action. Au risque encore de caricaturer, je dirais qu'avec Sartre, cet ensemble est relativement transparent et maîtrisable. Pour Lacan, Foucault, Certeau, Deleuze et d'autres, cet ensemble est traversé d'ombres et de lumières, il n'est que modérément maîtrisable. Il est excessivement complexe. Corrélativement, les technologies du sujet, de son corps et de ses objets participent de cette complexité.

La psychanalyse freudienne est au centre du débat. Elle souligne que le sujet est divisé entre une partie spécularisable et une autre - nonspécularisable - (dixit Lacan), c'est-à-dire entre une partie de lui-même dont le sujet perçoit l'image, et une partie de lui-même dont rien ne lui renvoie l'image et qu'il ne perçoit pas. Dans des termes plus freudiens que lacaniens, disons que je suis divisé entre le conscient d'une part, et le refoulé qui est en moi mais qui n'est pas moi d'autre part. Les sciences de l'homme et de la société ont abondamment illustré le fait que le sujet est soumis à des déterminations internes et externes, qu'il est mal armé pour percevoir, analyser, et encore moins contrôler. On comprend l'importance de ce débat si l'on se souvient que le sujet est un corps et a un corps. La manière dont on conceptualise le corps dépend des termes dans lesquels on pose ce débat.

Transvasement de la sève de raphia qui, après fermentation, donnera du vin de raphia (Mankon, 2002) 
largement une "sociologie sans les objets ». Le « sujet », par contre, est à la fois le sujet de droits et de devoirs pour le juriste en tant que personne physique, le sujet du symptôme corporel pour le médecin, le sujet constituant son monde pour le philosophe, le sujet assujetti à une souveraineté pour le politiste, le sujet de ses énonciations pour le linguiste et le sujet obscur et divisé pour le psychanalyste.

\section{La culture matérielle, essentielle au corps et à I'humain}

Pour ma part, je me situe sans hésitation dans une vaste tradition qui place le corps au cour de la subjectivité. Cette tradition est polymorphe. On y trouve celle qui va de Husserl à Berthoz et Petit (2006) en passant par Merleau-Ponty, tout comme celle qui va de Canguilhem à Foucault, ou celle de la psychanalyse. Comme le font remarquer Berthoz et Petit (2006: 19-24 et 139), on est ici très loin de la philosophie analytique tout comme de Wittgenstein, peu en prise sur le corps et les matérialités.

La tradition de Berthoz et Petit articule la physiologie de l'action à une réflexion sur l'intentionnalité et les kinesthèses. Les neurosciences cognitives sont à la base de leur réflexion. Ces deux auteurs (2006: 208-214) ont écrit des pages étonnantes pour montrer comment les kinesthèses, et par conséquent le corps propre, sont en prise avec la
Libation de vin de raphia devant un orchestre

« Le cerveau humain, tel quill est construit chez Homo sapiens, n'existe pas et n'existerait pas sans une interaction constante et essentielle avec les objets et les matérialités dans le mouvement » (Mankon, 1973).

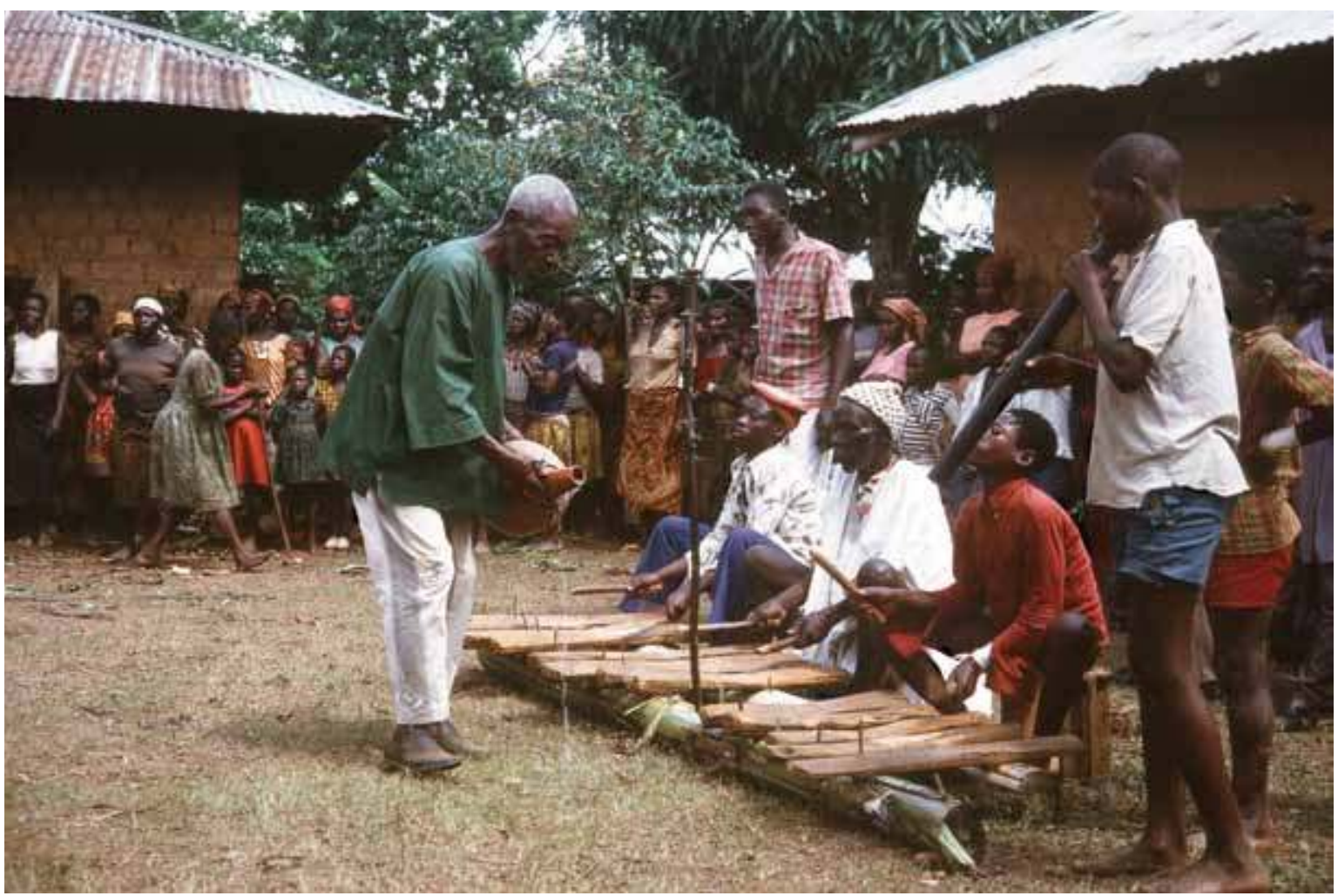


culture matérielle (qu'ils ne nomment pas comme telle cependant) de manière essentielle, c'est-à-dire que le cerveau humain, tel qu'il est construit chez Homo sapiens n'existe pas et n'existerait pas sans une interaction constante et essentielle avec les objets et les matérialités dans le mouvement ${ }^{3}$. Une simple observation naïve d'êtres humains en mouvement, permet de les voir en prise avec des claviers d'ordinateur, des équipements de cuisine, du mobilier domestique et urbain, leurs vêtements et accessoires, les outils de travail, etc. Lapport des neurosciences cognitives et de la physiologie du cerveau est essentiel à cet égard. La découverte du « système miroir » de Rizzolatti et Sinigaglia (2008) vient en renfort de l'affirmation du rapport essentiel de l'humain à la culture matérielle, en rajoutant une dimension de co-motricité et d'interaction entre sujets dans le mouvement, en rapport avec des matérialités. La ligne d'investigation qui part de Freud et qui passe par Lacan tient que le sujet n'est tel que d'être divisé et de rien d'autre (en rupture radicale avec la conception ontologique et substantielle de Sartre). Pour les chercheurs qui, comme c'est mon cas, n'ont qu'une maîtrise limitée du vocabulaire lacanien, on peut exprimer cette division en termes de clivage entre le moi conscient (en gros le cogito de Descartes) et le pré-conscient (ou le « ça ») comme refoulé, qui est avec moi mais qui n'est pas moi. Or, cette division advient ou échoue à advenir dans l'ontogenèse du sujet. Elle est contingente. Le processus est susceptible de tourner court. Il existe des humains chez qui le moi ne s'est pas séparé du refoulé. Ils ont l'inconscient à ciel ouvert. Chez eux, le sujet n'est pas advenu et ils sont incapables d'identifications. Ils ne sont ni dans le signifiant, ni dans le corps, ni dans la « culture matérielle ». Même pour les humains chez qui la division est advenue, le sujet n'est jamais là où il croit être. On ne peut donc pas ontologiser le sujet ni la subjectivité. C'est du contingent, mais un contingent qui fait toute la différence, en particulier

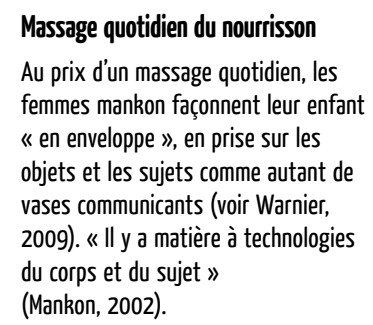

Massage quotidien du nourrisson

Au prix d'un massage quotidien, les « en enveloppe », en prise sur les objets et les sujets comme autant de vases communicants (voir Warnier, du corps et du sujet » (Mankon, 2002).

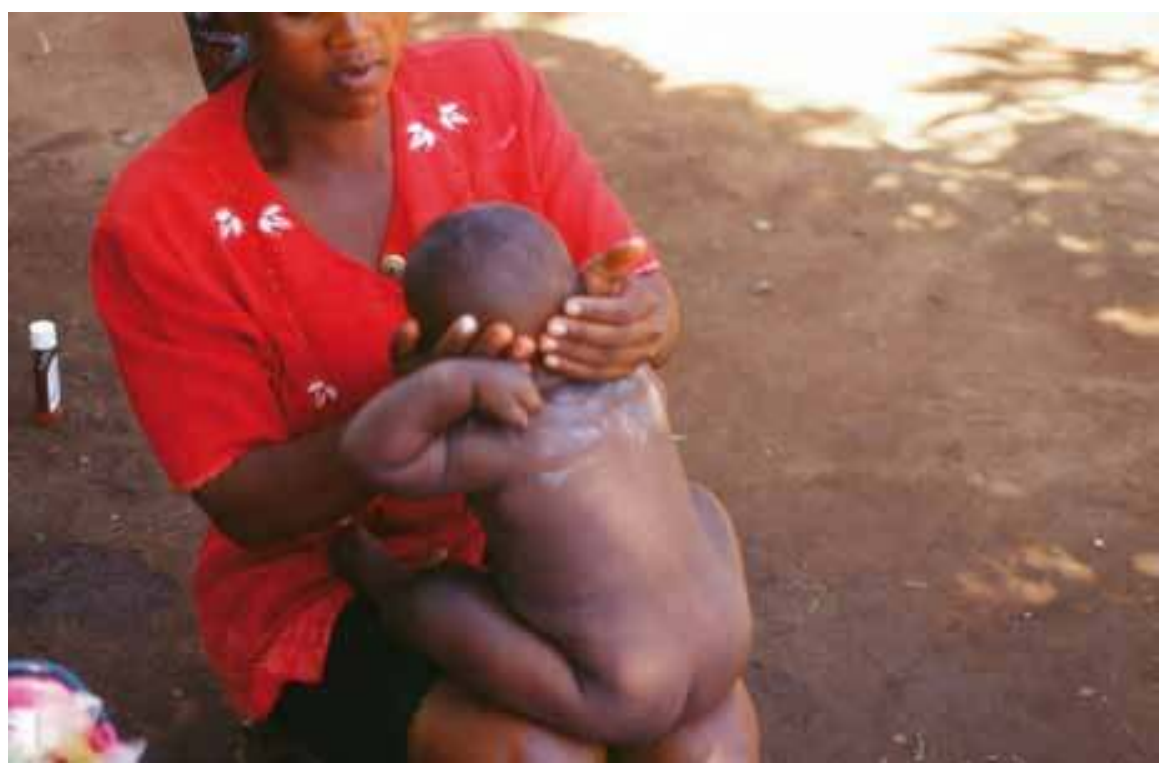


entre la pensée de Foucault et celle de Luc Ferry. En effet, si le sujet et son corps peuvent advenir ou devenir ceci plutôt que cela, ils font forcément l'objet d'un travail de construction et de façonnage. La subjectivité et le corps, ça se travaille. Ce sont des objets techniques. Il y a matière à technologies du corps et du sujet. Dans la tradition de Lacan, on peut citer le philosophe et psychanalyste S. Zizek (2007) qui se réclame de Hegel et qui souligne à juste titre la radicalité du clivage dans l'Occident contemporain entre une philosophie du sujet informée par les sciences de l'homme et de la société et une philosophie néolibérale de l'individu, hostile à la psychanalyse et aux sciences de l'homme et de la société. Ces dernières, en effet, ont pour ambition d'explorer l'humain, ce qui suppose de dépasser la simple ingénierie sociale organisant des « individus » prêts à l'emploi, dans laquelle l'idéologie néolibérale voudrait cantonner l'usage des sciences de l'homme et de la société. Je résume: le sujet est un humain individualisé par un corps vivant, investi par son désir et celui des autres. Bien qu'il ait un corps et qu'il soit un corps, le sujet n'est pas un donné de nature. Il est engagé dans un devenir (ou une genèse) qui peut échouer ou réussir à produire une subjectivité. Il est contingent. Il peut advenir ou ne pas advenir. N'étant jamais intégralement donné ni programmé au point de départ, le sujet doit être produit, façonné, configuré, et identifié dans son corps et son psychisme, en relation avec le monde qu'il constitue, ce qui ouvre un vaste domaine de déploiement de techniques spécifiques.

Revenons à Foucault et à ce qu'il énonce des techniques de soi et des savoirs sur les techniques du sujet - des technologies du sujet visant à le produire, l'identifier, le configurer, l'assujettir, en promouvoir la vie, en ordonner ou en autoriser la destruction. Étant donné que ces technologies ont une action sur les sujets, elles coïncident avec des technologies du pouvoir, que Foucault en est venu à concevoir comme plus diffus et productif que contraignant.

Enfin, les technologies du pouvoir reposent sur des « dispositifs » largement (mais pas seulement) matériels. En effet, ils sont également configurés par des idéologies spécifiques, des modes de production, des événements et des mouvements historiques. Les technologies du sujet s'adressent au corps, là où le sujet se prend lui-même pour objet de ses propres actions dans le « gouvernement de soi », ce qui implique l'usage de matérialités à titre essentiel. Foucault n’a ni nommé ni théorisé la « culture matérielle ». Mais sa philosophie du sujet est compatible avec (voire accueillante à) une prise en compte de la culture matérielle et des techniques du corps en rapport avec des « gouvernementalités subjectivantes». Dernière précision: Foucault amalgame deux processus distincts sous le même vocable de « subjectivation » : l'ontogenèse du sujet par division, et les identifications du moi lorsque celui-ci adopte des propriétés et configurations fournies pas son entourage, par les techniques de soi et par des innovations qui lui sont propres.
Fabrication et emballage de gruau de colocasia et de bananes douces

« Le travail sur les enveloppes et les contenus fait office de technologies du sujet. Celles-ci s'adressent au corps, là où le sujet se prend lui-même pour objet de ses propres actions, ce qui implique l'usage de matérialités à titre essentiel » (Mankon, 2002).

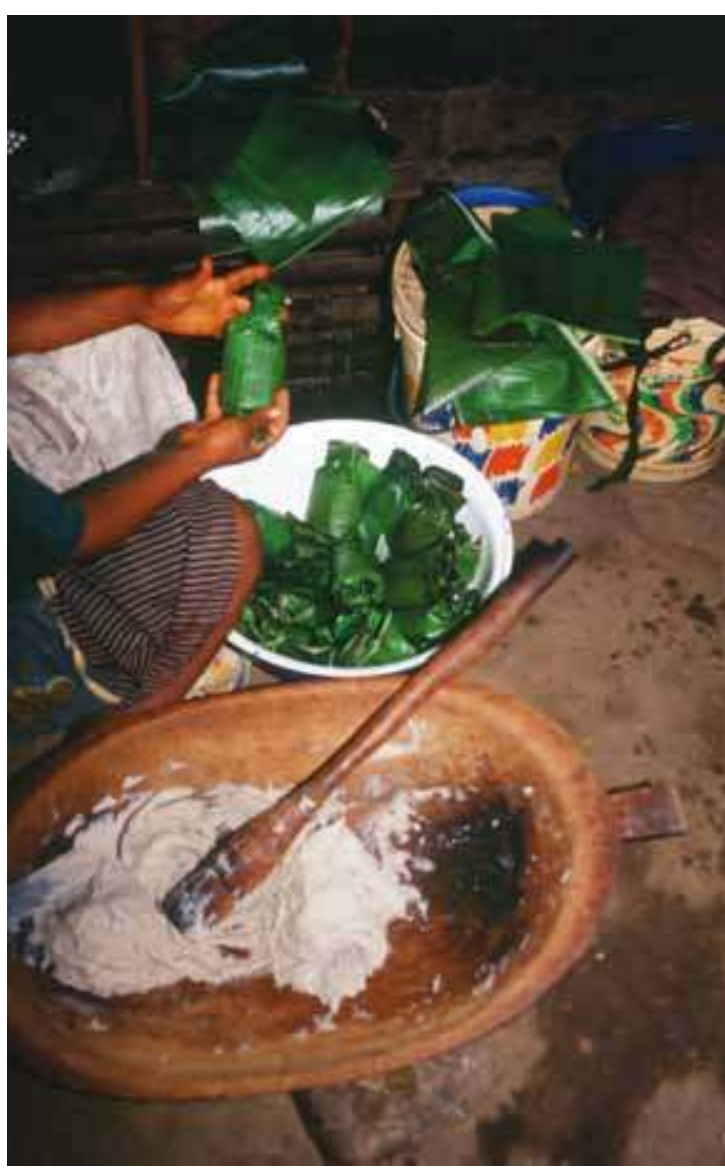




\section{Négociants en vin de raphia}

« Si le sujet opère une synthèse avec ses objets dans l'action, on ne voit guère comment système technique et société des sujets pourraient être distincts l'un de l'autre sinon à titre analytique » (Mankon, 1972)

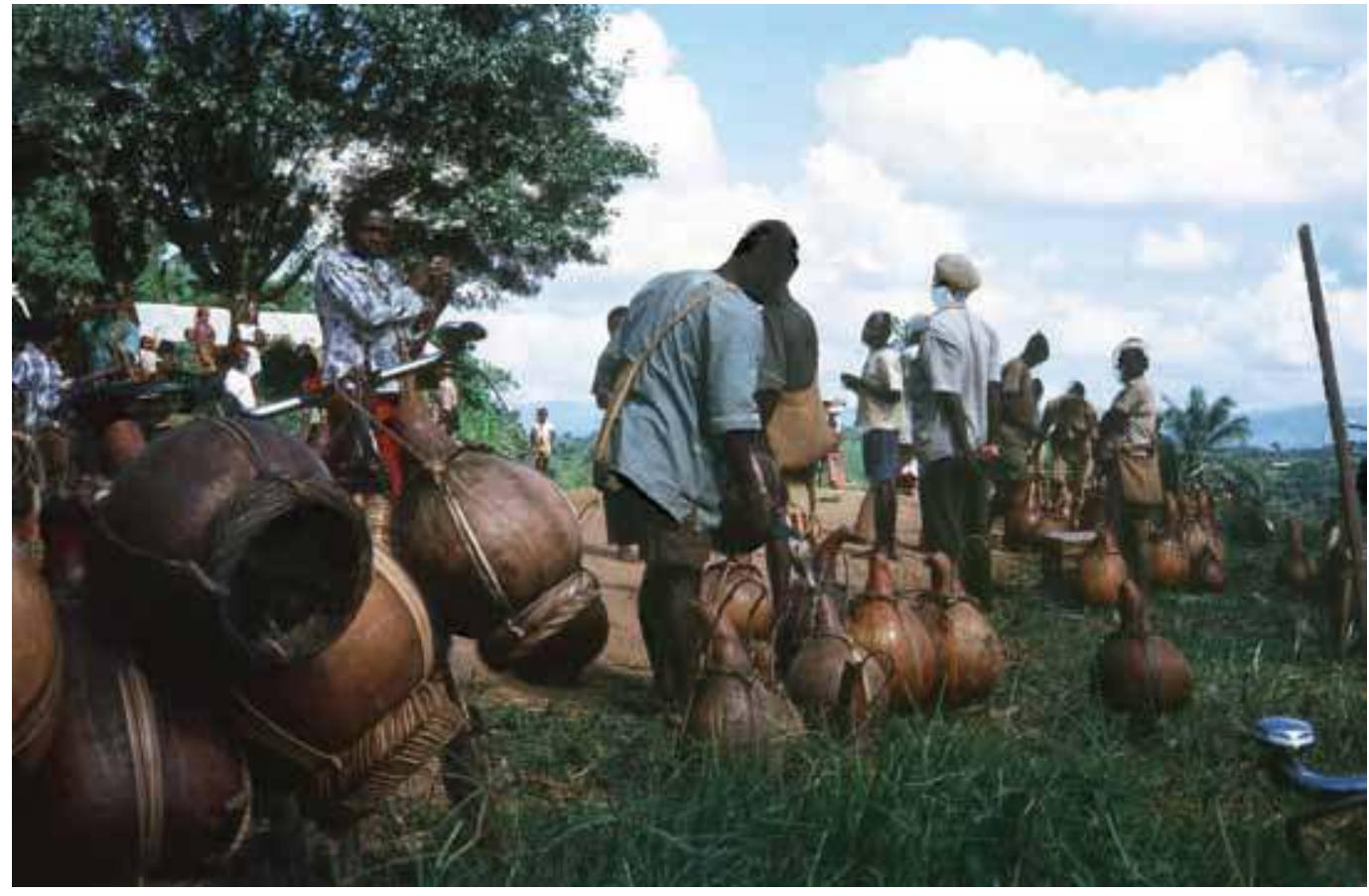

Prise en commun de gruau de colocasia et de bananes

« II est difficile d'introduire une distinction autre qu'analytique entre les systèmes techniques de la matière et les systèmes techniques du sujet » (Mankon, 2002)

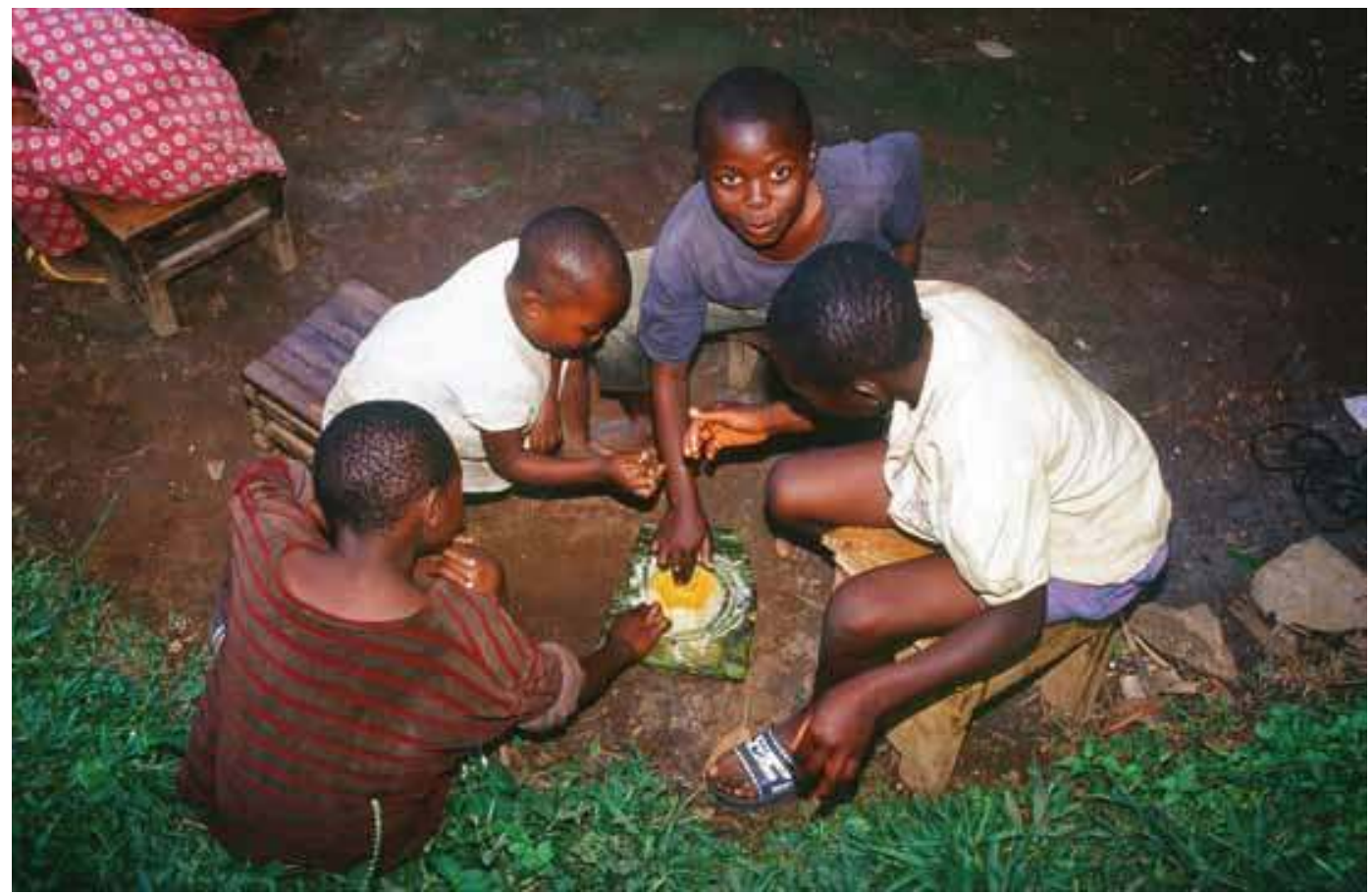




\section{Technologies du sujet et systèmes techniques}

Dans une perspective foucaldienne, les technologies du sujet sont articulées à ce qu'on appelle les « systèmes techniques » (voir Lemonnier 1986). Deux exemples: les techniques de surveillance et de punition sont étroitement liées aux innovations techniques de l'emprisonnement (bâtiments, optiques, miradors, armes, clôtures électriques, vidéosurveillance, biométrie, télédétection, etc.), elles-mêmes liées à des techniques qui se déploient en réseaux dans tout l'ensemble du système technique d'une société donnée. Deuxième exemple: le passage à un « bio pouvoir » est étroitement corrélé à toutes les techniques médicales, elles-mêmes en constante interaction avec des innovations dans des domaines proches, comme ceux des sciences de la matière et de la vie appliquées aux techniques médicales, mais aussi à des technologies organisationnelles dans les institutions de santé.

Je n'aborderai pas ici la question de la causalité, sauf à dire qu'après avoir fait couler des fleuves d'encre, on peut s'accorder pour dire que le déterminisme technique est discrédité, comme son alter ego qu'est le déterminisme social, tant les innovations techniques résultent souvent de dynamiques sociales, économiques, et, en bout de course, du corps propre et des kinesthèses des chercheurs et des sujets innovants qui ne sont pas de purs cerveaux en apesanteur, mais toujours des sujets-avec-leurs-objetsincorporés. Si le sujet opère une synthèse avec ses objets, on ne voit guère comment systèmes techniques et société pourraient être distincts l'un de l'autre et en vis-à-vis.

\section{Mouvement, incorporation, désincorporation}

Il résulte des remarques précédentes qu'il est difficile d'introduire une distinction autre qu'analytique entre les systèmes techniques de la matière et les systèmes techniques du sujet. Au point où j'en suis de mes réflexions, je verrais bien un système technique en quelque sorte « unique », avec des boucles d'action et de rétroaction, des artefacts aux sujets, canalisées par des savoirs, des savoir-faire, des innovations idéologiques et religieuses, des relations de production, des conflits, alliances et dynamiques historiques.

L'artefact matériel est un des pôles de ces actions/rétroactions. Mais il faut insister sur le fait que, dès lors qu'on place le mouvement et les kinesthèses au cœur des techniques, l'artefact ne dispose d'aucune autonomie par rapport au sujet. Il m'importe ici de souligner une distinction qui me semble essentielle entre l'artefact pris pour sa valeur-signe dans un système de communication ou de connotation (comme l'ont illustré Barthes, Lévi-Strauss, Baudrillard et de nombreux autres analystes), et l'artefact pris pour sa valeur praxique dans le mouvement et dans un système d'action (comme c'est le cas chez Berthoz, Petit, Rizzolatti, Foucault, etc.). Dans le 
Maison

« J'entre dans la maison

la maison devient mon prolongement. Je l'habite.

Je la quitte, la maison devient un objet extérieur »

(Esu, Cameroun, 1984). premier cas, l'artefact « vitrinifié », comme truchement des significations ou quasi-mot, est hors mouvement. Il dispose d'une certaine autonomie par rapport au sujet communiquant, à l'instar de la parole parlée ou de l'énoncé, relativement autonome par rapport à la parole parlante ou à l'acte d'énonciation. Il peut être analysé comme un signe, fait d'un signifiant (l'objet) et du signifié qui s'attache à lui. Par exemple, la cravate comme signifiant connote la masculinité et le statut social, qu'elle soit exposée à la vente dans une vitrine ou portée par un homme (ou une femme qui signale alors qu'elle fait « comme les garçons »). Au regard de sa fonction d'objetsigne, le mouvement lui est inessentiel. Celui-ci n'ajoute ni ne retranche rien à la signification. Le système des objets signifiants est informé par des codes que le sémiologue s'attachera à analyser. Il peut le faire pour l'essentiel hors mouvement.

Par contre, dès lors qu'on prend en compte la valeur praxique de l'artefact, c'est-à-dire comment le sujet est en prise avec l'objet et ce qu'il fait avec, on est ipso facto situé dans un monde en mouvement où le corps vivant, la matière, les artefacts et les sujets bougent. Au demeurant, aucun sujet ne peut se mouvoir sans mobiliser des kinesthèses en prise essentielle avec des

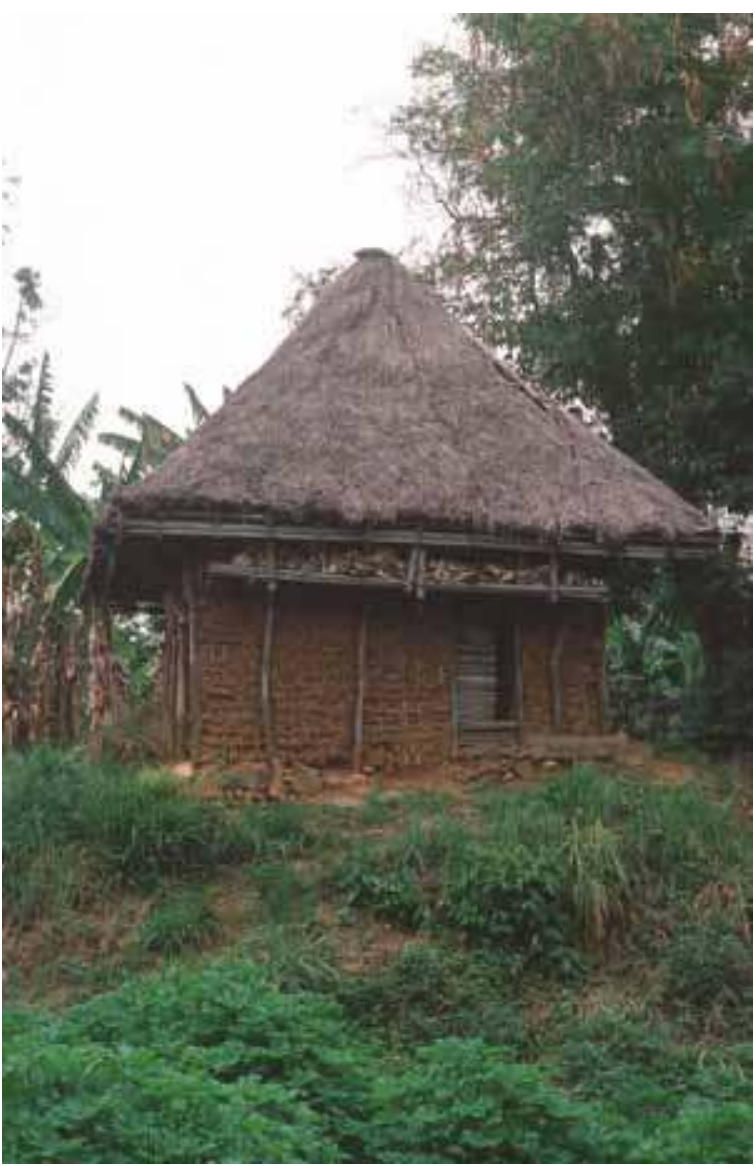


matérialités. Les artefacts ne jouissent d'aucune autonomie de mouvement par rapport au sujet, et réciproquement. Bien entendu, ce point mériterait discussion tant il suscite aussitôt mille objections tirées du domaine de la robotique. Drones, automates, machines-outils et robots ne sont-ils pas autant d'exceptions à ce que je viens d'énoncer? De plus, Bruno Latour a vu dans les objets matériels des acteurs à part entière - point de vue que je ne partage pas. Sans vouloir botter en touche, j'enregistre l'existence de ces problèmes et j'en ajourne la discussion afin de ne pas dépasser les limites raisonnables d'un article de synthèse.

Revenons à la ligne principale du raisonnement: dans le mouvement, la mise en œuvre des technologies du sujet et celle des technologies des artefacts sont très largement confondues. Il me semble que l'ergonomie et les théories de la connaissance distribuée et de l'action située vont dans le sens de cette affirmation, tout comme ce qu'énoncent Berthoz et Petit (2006 : 212) sur la physiologie et la phénoménologie de l'action par rapport aux objets. Qu'on en juge:

« Le corps propre fonctionne comme un échangeur du subjectif en objectif, et réciproquement. Je monte dans ma voiture: la voiture devient mon prolongement. J'en descends, la voiture redevient un objet extérieur. Le corps propre, cet objet curieux, va tirer parti de son statut spécial pour, à un moment donné, assimiler à soi-même un objet du monde extérieur, et ainsi donner à cette relation entre le sujet et le monde une valeur de connaissance par l'action, par la praxis».

Le point de convergence des technologies du sujet et de celles des artefacts peut être localisé grâce à la métaphore de l'incorporation et de la désincorporation. Dans le mouvement, sujet et objet matériel ne font qu'un. Les connaissances procédurales et l'investissement libidinal dans les objets, déjà soulignés par Schilder dès 1923 (cf. 1935), sont tels qu'un sujet qui conduit une voiture ne fait qu'un avec celle-ci. C'est un « sujetvoiture » dont « l'image du corps » (alias schéma corporel, corps propre, kinesthèses, algorithmes moteurs, selon que l'on parle "schildérien », « merleau-pontien », « husserlien » ou « parlebasien $»^{5}$ ) s'est dilatée jusqu'à englober la voiture, si bien que, par exemple, le conducteur perçoit la prise sur la route à l'interface des pneus et de l'asphalte et non à l'interface de ses mains et du volant. Précisons que la « praxéologie motrice » de Pierre Parlebas (1999), inspirée de Lespinas, de Mauss, de divers courants de la psychologie cognitive et de la théorie des jeux, propose une batterie de concepts destinés à analyser les pratiques ludiques et sportives à partir d'une prise en compte de la motricité qui donne le monisme tant recherché et si rarement atteint. Elle a considérablement inspiré les travaux du groupe « Matière à Penser » dont il sera question plus bas.

Ce processus d'incorporation, qui se fait dès lors que le sujet a acquis par apprentissage les connaissances procédurales adéquates et qu'il est en prise avec l'artefact dont il fait usage, a pour contrepartie un processus de désincorporation lorsque le sujet abandonne l'artefact « $\mathrm{Al} »$ (une voiture), pour un artefact « $\mathrm{A} 2$ » (le parking et l'ascenseur de l'entreprise), puis pour 


\section{Forgeron au travail}

« Techniques de soi et techniques métallurgiques sont pratiquement confondues, tant, dans cette société, le fer est perçu comme un danger et doit être produit et manipulé par des êtres entièrement pacifiés » (We, Cameroun, 1984)

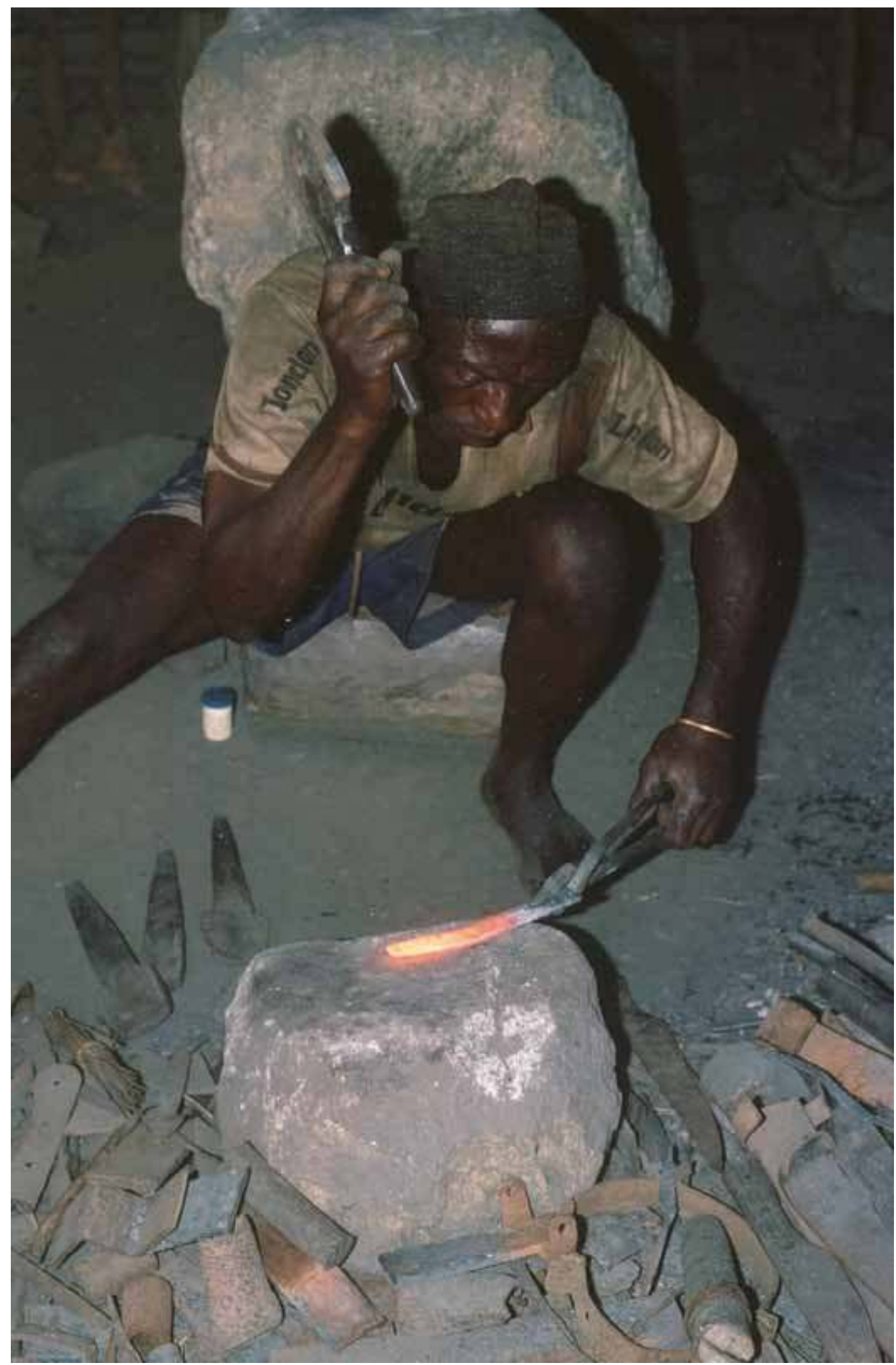


un artefact « A3 » (le robot de construction automobile qu'il pilote). Dans l'incorporation/désincorporation, chaque artefact singulier est contingent pour le sujet, mais leur ensemble, à titre de "culture matérielle », est essentiel au sujet, à son action, et à la condition humaine, au même titre que le langage.

\section{Mise en œuvre du paradigme et perspectives de recherche}

Ce paradigme théorique s'est construit, et continue de l'être, au sein du réseau « Matière à Penser » (MàP ${ }^{6}$ et de ses prolongements rhizomatiques. Il soutient un programme d'ethnographie très concrète sur les mouvements, les choses, les corps, les pratiques matérielles. J'ai contribué à sa construction afin de disposer d'une boîte à outils théorique me permettant d'analyser les technologies du sujet et du pouvoir dans un royaume africain au temps du " retour des rois » sur le devant de la scène politique africaine (voir Warnier $1999,2004,2005,2007,2009)$. À mon sens, seule la formule scientifique de la monographie (plutôt que de courts articles théoriques) est à même de valider un tel paradigme, car elle possède une exigence de complétude, à défaut de totalisation. Pour être convaincante, elle ne doit pas laisser de résidu empirique en dehors de son périmètre, tel qu'il nécessiterait la mise en œuvre d'un paradigme alternatif. J'ai donc proposé une monographie de ce royaume qui mobilise une ethnographie des technologies du sujet et du pouvoir étayée sur une culture matérielle spécifique dont l'usage est attesté sur toute la hauteur de la hiérarchie sociale, du roi au cadet célibataire, et qui fait que ce royaume emporte l'adhésion de ses sujets et a pu produire une hégémonie sur plusieurs siècles en dépit d'extrêmes inégalités sociales et de conflits répétés.

Je suis redevable à Pierre Lemonnier ${ }^{\top}$ d'avoir posé une question qui ouvre de nouvelles perspectives de recherche: dans ce royaume, comment les technologies du sujet s'articulent-elles avec le système technique? J'ai repris cette question à mon compte dans le présent article. Elle est d'autant plus pertinente que, comme je le pense, technologies du sujet et technologies des artefacts font système. N'ayant guère enquêté en détail sur les systèmes techniques locaux, sauf dans le cas de la métallurgie (fonte et forge), je suis mal équipé pour y répondre. Dans le cas de la métallurgie, ainsi que je l'ai montré (voir Warnier 2004), techniques de soi et techniques métallurgiques sont pratiquement confondues, tant, dans cette société du Cameroun, le fer est perçu comme un danger et doit être produit et manipulé par des êtres entièrement pacifiés. Cette investigation devrait être étendue aux domaines de l'agriculture, de l'élevage, du bâtiment, de la boissellerie, etc.

Julien Bonhomme a pointé du doigt un deuxième domaine qui mérite investigation. Ce domaine concerne la marge de manœuvre des sujets individuels par rapport aux techniques de soi « proposées voire imposées » aux individus, comme l'écrivait Foucault (1989) et par rapport aux 
Onction d'huile de palme sur une calebasse

« Les sujets ne sont pas faits du même bois selon qu'ils ont incorporé la culture matérielle d'un agriculteur Mankon ou d'un cadre supérieur français » (Mankon, 1973).

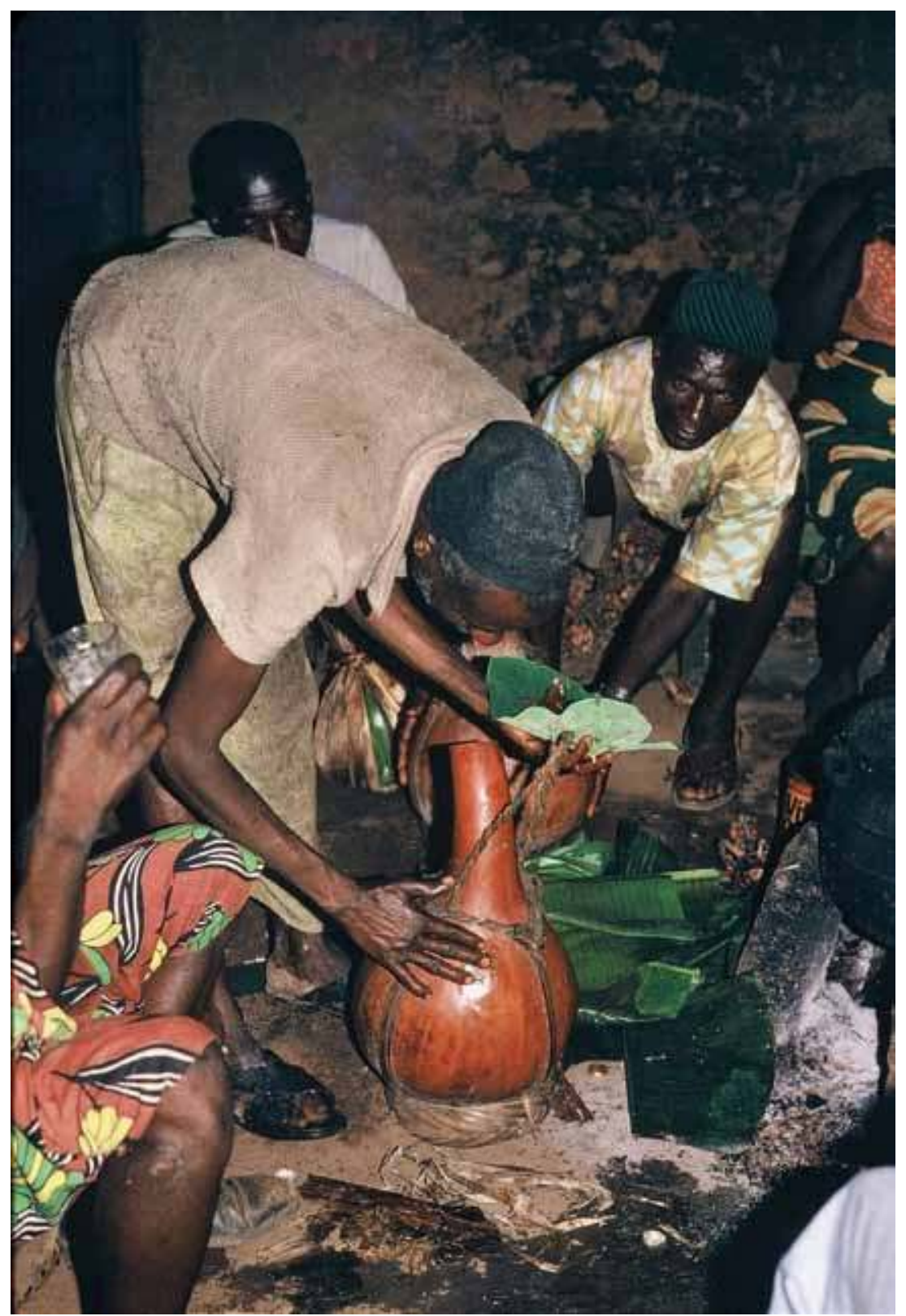

gouvernementalités subjectivantes. Je pourrais ajouter: la « gouvernementalité » n'est-elle pas un cache-misère de la «structure » qui dissoudrait l'historicité du sujet et du royaume? À mon avis, seuls des récits de vie d'un certain nombre de sujets du royaume (celui du roi vient de faire l'objet d'une publication: Fo Angwafo 2009) livreraient des données empiriques permettant d'argumenter sur ce point. En sous-œuvre de cette question relative aux données de terrain se trouve une question plus philosophique. 
C'est celle de la liberté du sujet dans un cadre foucaldien ou dans celui d'une « physiologie et phénoménologie » de l'action. Il est certain qu'une telle question reçoit une réponse beaucoup plus complexe et nuancée dans le cadre de ces paradigmes que dans celui d'une philosophie sartrienne du sujet, ou néolibérale de l'individu. Dès lors en effet que c'est du sujet qu'il s'agit, avec ses investissements libidinaux, son inconscient, son corps, sa culture matérielle, son système technique - le tout en interaction avec d'autres sujets - (mais aussi avec son quant-à-soi, ses choix, ses refus, son intelligence et sa part de conscience de soi et des autres), la réponse ne peut pas se réduire à quelques formules à l'emporte-pièce. Dire qu'un sujet se fabrique par la mise en œuvre de technologies spécifiques qui en font un sujet-et-ses-objets dans l'action revient à dire que les sujets ne sont pas faits du même bois selon qu'ils ont incorporé la culture matérielle d'un Pygmée baka ou d'un cadre supérieur français.

\section{Technologies du sujet, technologie culturelle et consommation}

Le point de vue développé dans cet article vise à expliciter un certain nombre des présupposés philosophiques des travaux ethnologiques du groupe « Matière à Penser ». Ce n'est pas un programme de recherche à venir. Le groupe a une œuvre derrière lui: sur la fabrication de meubles chinois et des citoyens de la République à Paris (M.-P. Julien 2009), sur « habiter une pièce » (C. Rosselin 2009), sur les égouts de Montpellier (A. Jeanjean 2009), sur les Hauts de la Réunion (F. Hoarau 2009), etc. Il considère le rapport praxique à la culture matérielle comme une propriété essentielle du sujet, et le sujet non pas comme un donné, mais comme l'objet d'une fabrication. Il en résulte que les technologies du sujet et les technologies des choses sont étroitement imbriquées. Il y a donc « matière à penser », et cela se décline: matière à politique, à religion, à violence, etc.

Comment cette approche s'articule-t-elle à la technologie culturelle et à l'anthropologie des techniques à la française? Une fois encore sans faire dans la dentelle, je dirais que les deux domaines de l'anthropologie que je viens d'évoquer ont pu faire les avancées remarquables que l'on connaît en traçant rigoureusement le périmètre de leur objet grâce à une définition maussienne des techniques comme « actions traditionnelles et efficaces » sur la matière, dans les activités de travail. Par définition, l'action prise en compte était l'action transitive du travailleur qui s'applique à la matière. Les notions de chaîne opératoire, de système et de geste techniques, d'outil, etc. braquaient les projecteurs sur les processus de façonnage de la matière. Seuls les savoir-faire attiraient l'attention sur l'agent lui-même, mais en tant que travailleur plutôt qu'en tant que sujet. Ce que le groupe MàP a proposé consiste à élargir le propos en considérant que les techniques, en tant qu'actions « traditionnelles et efficaces » s'appliquent symétriquement 
Pot de médecines enfoui sous un four à réduction de même forme et posé sur deux morceaux de tuyère

« Il y a matière à symboles, à politique, à religion... »

(Bakwang, Cameroun, 1976). à la matière et au sujet de l'action. De ce fait, le domaine d'étude privilégié n'était plus les activités de travail, mais la vie quotidienne et les rapports politiques. Le risque de cette démarche étant évidemment de perdre en rigueur et en cohérence ce qu'elle gagnait en compréhension. Enfin, comment cette approche s'articule-t-elle aux études de consommation, à l'histoire, à la phénoménologie et à la sémiologie des choses telles qu'elles ont été développées avec tant de succès en Grande-Bretagne? Il me semble que les objectifs sont proches les uns des autres. Pour ne prendre qu'un exemple, la notion d'objectification développée par D. Miller rejoint celle d'incorporation. La différence principale, me semble-t-il, vient des outillages conceptuels qui sont mis en œuvre de part et d'autre. Peu à peu, les approches du groupe MàP ont mobilisé les apports de la praxéologie, des sciences du vivant, des neurosciences cognitives, et des thématiques foucaldiennes des technologies du sujet et de l'assujettissement, relativement peu mobilisés outre-Manche. Dans la mesure où aucun programme scientifique ne peut tout embrasser, il est clair que l'avancée de la recherche se paye de choix d'objectifs et de moyens qui se complètent les uns les autres.

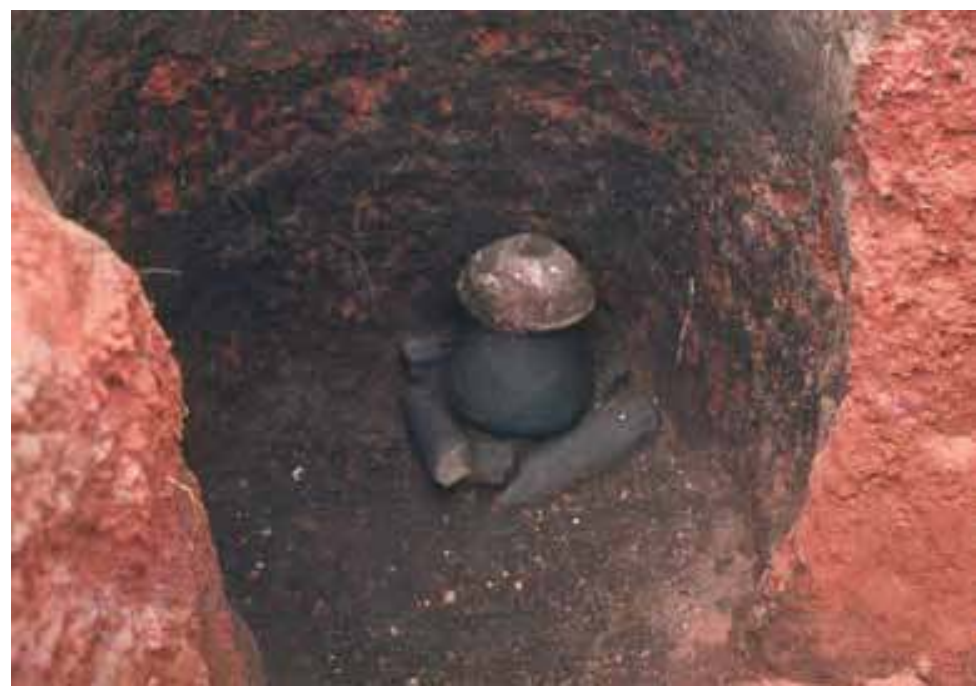




\section{NOTES}

Toutes les photos sont de l'auteur. Photographie d'ouverture : « Massage quotidien du nourrisson » Le corps n'est constitué comme tel qu'en étant investi par un sujet qui est et qui a un corps. (Mankon, Cameroun, 2002).

1. La phénoménologie, déjà esquissée par Hegel, fut considérablement développée par Edmund Husserl (1859-1938). Au risque de caricaturer, la question centrale qu'il pose est la suivante: comment le sens vient-il au monde lorsqu'il n'est plus garanti par une transcendance? La réponse est que le sens vient au monde dans l'interaction entre le sujet et le monde qu'il constitue. Or le sujet « est au monde » par son corps. D'où la centralité du corps, des kinesthèses et de la constitution du monde par le corps propre dans la phénoménologie de Husserl et chez ses successeurs.

2. Voir note $n^{\circ} 1$.
3. Sur le fait que le fonctionnement du cerveau ne s'explique que par rapport à un sujet en mouvement, voir Berthoz (1997).

4. Sur la praxéologie motrice de Pierre Parlebas, voir Parlebas (1999).

5. Ce réseau s'est constitué en groupe de travail pendant une petite quinzaine d'années, d'environ 1992 à 2006. Les membres de ce groupe ont publié un certain nombre d'ouvrages dont les plus emblématiques sont Julien et Warnier (éds., 1999), Julien et Rosselin (2005), Julien et Rosselin (éds., 2009), Warnier (2009).

6. Lors d'une présentation de ma monographie au musée du Quai Branly en avril 2009.

7. Lors de la même présentation au musée du Quai Branly. 


\section{RÉFÉRENCES}

Andrieu, Bernard, 2006, Le Dictionnaire du corps en sciences humaines et sociales. Paris, CNRS Éditions.

Audier, Serge, 2008, La Pensée anti-68. Essai sur les origines d'une restauration intellectuelle. Paris, La Découverte.

Berthelot, Jean-Michel, 1988, "Le Discours sociologique et le corps », Quel corps? n 34-35, mai 1988: 72-83.

Berthoz, Alain 1997, Le Sens du mouvement. Paris, Odile Jacob.

Berthoz, Alain \& Petit, J.-L., 2006, Phénoménologie et physiologie de l'action. Paris, Odile Jacob.

Blandin Bernard, La Construction du social par les objets. Paris, PUF.

Csordas, Thomas éd., 1994, Embodiment and Experience. The Existential Ground of Culture and Self. Cambridge, Cambridge University Press.

Featherstone, M., Hepworth, M. \& Turner, B éds., 1991, The Body: Social Process and Cultural Theory. Londres, Sage.

Ferry, Luc \& Renaut, Alain, 1985, La Pensée 68. Essai sur l'anti-humanisme contemporain. Paris, Gallimard.

Fo Angwafo III, 2009, Royalty and Politics. The Story of My Life. Mankon. Bamenda, Langaa RPCIG.

Foucault, Michel, 1989, Résumé des cours 1970-1982. Paris, Gallimard.

Julien, Marie-Pierre \& Rosselin, Céline, 2005, La Culture matérielle. Paris, La Découverte, (coll. « Repères »).

Julien, Marie-Pierre \& Rosselin, Céline, 2009, Le Sujet contre les objets... tout contre. Ethnographies de cultures matérielles. Paris, CTHS.

Julien, Marie-Pierre \& Warnier, Jean-Pierre, 1999, Approches de la culture matérielle. Corps à corps avec l'objet. Paris, L'Harmattan.

Le Breton, David, 1990, Anthropologie du corps et modernité. Paris, PUF.

Lemonnier, Pierre, 1986, «The Study of Material Culture Today: Toward and Anthropology of technical System », Journal of Anthropological Archaelogy, 5 (2): 147 - 186.

Marsano, Michela, 2007, Dictionnaire du corps. Paris, PUF.

Mauss, Marcel, 1936, « Les Techniques du corps », Journal de psychologie, Vol. xxxII, n 3-4, (reproduit dans Sociologie et anthropologie. Paris, PUF, 1950 : 331-362).

Parlebas, Pierre, 1999, Jeux, sports et sociétés. Lexique de praxéologie motrice. Paris, INSEP.

Rizzolatti, Giacomo \& Sinigaglia, Corrado, 2008, Les Neurones miroirs. Paris, Odile Jacob.

Schilder, Paul, 1935, The Image and Appearance of the Human Body; Studies in the Construcive Energy of the Psyche. Londres, Kegan Paul (trad. fr. : L'Image du corps. Paris, Gallimard, 1968).

Warnier, Jean-Pierre, 1999, Construire la culture matérielle. L’homme qui pensait avec ses doigts. Paris, PUF, (Coll. « Sciences sociales et société »).

Warnier, Jean-Pierre, 2004, «Métallurgie ancienne, identifications et domestication de la violence au Cameroun », in J.-F. Bayart \& J.-P. Warnier éds., Matière à politique. Le pouvoir, les corps et les choses, Paris, CERI-Karthala : 181-194, (Coll. « Recherches internationales »).

Warnier, Jean-Pierre, 2005, "Inside and Outside. Surfaces and Containers», in C. Tilley \& al. éds., Handbook of Material Culture. Londres, Sage: 186-202.

Warnier, Jean-Pierre, 2007, The Pot-King. The Body and Technologies of Power. Leiden, Boston, Brill.

Warnier, Jean-Pierre, 2009, Régner au Cameroun. Le Roi-pot. Paris, CERI-Karthala, (Coll. « Recherches internationales $»)$.

Zizek, Slavoj, 2007, Le Sujet qui fâche. Paris, Flammarion. 


\section{RÉSUMÉ}

Les Technologies du sujet: une approche ethno-philosophique. Il existe des techniques du corps, du pouvoir et du sujet, auxquelles correspondent des savoirs qui constituent autant de technologies. Ces technologies s'articulent avec celles des matières et des artefacts. Cela dit, dès lors qu'il est question du « sujet » plutôt que de « l'individu », un minimum de réflexion philosophique s'impose.

\section{ABSTRACT}

Technologies of the Subject: an Ethno-Philosophical Approach. There are techniques of the body, of power and of the subject which are the object of corresponding knowledge or technologies. These technologies are articulated to the technologies of artefacts and material things. However, given the fact that the « subject » rather than the « individual » is put on the agenda, a modicum of philosophical elaboration is required.

\section{MOTS CLÉS}

Corps, culture matérielle, individu, objet, phénoménologie, praxéologie, sujet, technologies.

\section{KEYWORDS}

Body, individual, material culture, object, phenomenology, praxeology, subject, technology. 